Revista de Investigación Lingüística, 22 (2019)

ISSN: 1139-1146

Universidad de Murcia

\title{
LAS CONSTRUCCIONES DE INDEFINICIÓN Y EL ROL DEL CONTEXTO
}

\author{
Liliana Ruiz Velasco D. \\ Universidad Intercultural del Estado de Puebla \\ rliliana_@hotmail.com
}

\begin{abstract}
Resumen: En trabajos previos hemos venido planteando la existencia de una construcción indefinida con DAR la gana. En este trabajo abordamos la relación que guarda con el contexto. Para ello partimos de la teoría de la construccionalización, como un proceso de cambio lingüístico y en lo particular aquel vinculado a la gramaticalización. En este sentido diversos trabajos (Heine, 2002; Diewald, 2006) han resaltado el rol del contexto. Se plantea que el significado de indefinición surge desde la construcción más antigua DAR gana y con la construcción DAR la gana se verifica su carácter polisémico por el que en algunos contextos pasa a primer plano un significado volitivo mientras que en otros es el significado de indefinición.
\end{abstract}

Palabras clave: construccionalización, indefinición, gramaticalización, historia del español

\begin{abstract}
Previous work has shown the existence of an indefinite construction with DAR la gana. The present paper deals with the relationship that this construction keeps with context. The point of departure is constructionalization theory, as a process of linguistic change, particularly the process of grammaticalization. Several authors (Heine, 2002; Diewald, 2006) have emphasized the role of context. It is shown that indefiniteness meaning appears with the older construction DAR gana, while with DAR la gana we found a polysemous meaning according to the context: in some contexts the volitional reading comes to the front while in others it is the indefinite reading.
\end{abstract}

Keywords: constructionalization, indefiniteness, grammaticalization, Spanish history 


\section{INTRODUCCIÓN}

En trabajos previos (Ruiz Velasco, en prensa) hemos venido señalando la existencia de una construcción indefinida basada en el esquema: NEXO SUBORDINANTE + EXPRESIÓN VERBAL DE DISPOSICIÓN MENTAL. Cuando hablamos de una expresión verbal de disposición mental nos referimos a verbos fundamentalmente de carácter volitivo como pueden ser QUERER O PREFERIR, pero también otros como GUSTAR / ANTOJÁRSELE / CONSIDERAR. Cuando hablamos de un nexo subordinante, nos referimos a aquellos que permiten elaborar construcciones de relativo como que, quien, donde, cuando, etc., los más prototípicos, pero también nexos más generales de carácter fundamentalmente adverbial como pueden ser porque, siempre que, según, etc. incluyendo en algunos casos el condicional si. Entre las expresiones de disposición mental se halla DAR la gana, de manera que encontramos un esquema general, del tipo NEXO SUBORDINANTE + DAR la gana, y uno más particular, del tipo HACER lo que DAR la gana, tomando como verbo prototípico HACER si bien puede emplearse otro tipo de verbos. En ambos casos tratamos con construcciones que permiten marcar la indefinición.

Desde un enfoque pragmático, que es el que asumimos en este trabajo, la definición remite a la identificabilidad de un referente único en un universo discursivo dado, esto es, el conocimiento de a qué elemento se está haciendo referencia, sea porque es percibido, mencionado o puede inferirse (Lyons, 1999; Leonetti, 1999: $\$ 12.1 .1 .3)$. La indefinición se refiere a la falta de identificabilidad del referente de manera unívoca, de manera que un elemento indefinido da la indicación al oyente de que el referente en cuestión no es identificable (Leonetti, 1999: § 12.2.1.2). En este sentido, se ha dicho que los pronombres de este tipo son de indistinción o indiferencia (Fernández Ramírez, 1987: \$197), o de elección libre para el oyente (Haspelmath, 1997: 48). En su trabajo sobre los pronombres indefinidos, Haspelmath (1997) señala que se dan series del tipo somebody, something, somewhere, sometime, etc. que se corresponden con cada una de las categorías ontológicas mayores de persona, cosa, lugar, tiempo, etc. Normalmente, la raíz indica la categoría ontológica mayor más un elemento formal que comparten los elementos de la serie (en el caso que nos ocupa: some-), al que llama un marcador de indefinición. No obstante, no siempre se trata de compuestos, pues en algunas lenguas pueden emplearse frases nominales o frases con una cláusula de relativo libre (ibid.: 52-55). En español, Fernández Ramírez (1987) señala que, además de las formas compuestas cualquier(a), comoquiera, quienquiera, dondequiera, se dan fórmulas indefinidas del tipo hay a sido el que haya sido (\$199). Las construcciones de indefinición a las que hemos hecho referencia son fórmulas similares a estas, pues tienen un carácter frasal y se encuen- 
tran menos gramaticalizadas que los pronombres indefinidos cualquiera, comoquiera, etcétera.

El presente trabajo se ocupa del rol del contexto. Ahora bien, cabe preguntarse si el significado de indefinición ya se halla codificado en la construcción o depende de cuestiones pragmáticas. Como veremos, la construcción ha mantenido un doble significado en el que a veces se perfila más un significado volitivo, como una disposición mental para algo, y en otras se perfila más el significado de indefinición. En lo que sigue hacemos primeramente una caracterización de la construcción DAR la gana, para luego pasar a abordar las nociones de cambio lingüístico y el rol del contexto, y enseguida presentar el análisis y las conclusiones.

\section{LA CONSTRUCCIÓN DAR LA GANA}

De acuerdo con los datos del Corpus diacrónico del español (CORDE, <http:// corpus.rae.es/cordenet.html>), la construcción DAR la gana se documenta por primera vez en el s. XVII. Esta forma parte de una familia de construcciones. En primera instancia surgió DAR gana en el s. XVI, a partir de la cual se desarrollan dos construcciones diferenciadas: DAR la gana, y DAR ganas, que aparece en el s. XVIII (Ruiz Velasco, 2017 y en prensa). Ambas han sido renovadas con otros elementos léxicos; así, decimos también: ENTRAR ganas y PEGÁRSELE la gana, y en español mexicano HINCHÁRSELE la gana. DAR la gana y DAR ganas se distinguen por una serie de características, en parte debido a una mayor fijación de DAR la gana (Ruiz Velasco, 2017), a saber:

- Dar la gana tiene un orden fijo sin que permita la anteposición de la frase nominal (la gana + DAR), salvo en usos creativos en los que el enunciado ya no forma parte de esta construcción

- Asimismo, requiere de un determinante definido y ha de estar en singular

- Los adjetivos que admite son de tipo intensificador, como pueden ser real/ puta/regalada/pinche/santísima, etc.

- Requiere fuertemente la presencia del clítico de objeto indirecto con el rol de experimentante

DAR la gana aparece primordialmente en dos tipos de construcciones distintas: una con la negación (No me da la gana) y las construcciones de indefinición a las que hemos hecho referencia. En estas últimas se dan también una serie de características (Ruiz Velasco, en prensa y ms.): 
- En principio, DAR la gana puede presentar la estructura: PRO.OI + DAR la gana + (de) $\mathrm{O}$, no obstante, puede emplearse con complementación nula: PRO.OI + DAR la gana $+\varnothing$. Esta es la estructura más frecuente. De hecho, en las construcciones indefinidas, se requiere de un sintagma escueto de ese tipo, sin complementos adjuntos y sin que el objeto indirecto aparezca como frase preposicional (p. ej. Yo hablo la lengua que me da la gana, s. XIX, Mariano José de Larra, El álbum).

- Aparecen prototípicamente en construcción de relativo, no obstante, a diferencia de lo señalado por Haspelmath (1997), pueden ser de distinto tipo: de relativo libre, con antecedente, semilibre o con un relativo compuesto o cuantificador; asimismo, encontramos la construcción con nexos subordinantes de carácter adverbial de distinto tipo (de causa, condición, tiempo y manera, p. ej. ... porque eso de la esquililla cada uno puede entenderlo conforme le dé la gana (s. XIX, Sebastián de Miñano, Sátiras y panfletos del Trienio Constitucional (18201823)).

- Se emplean prototípicamente en presente, ya sea de indicativo o subjuntivo, si bien se encuentran también en pasado y futuro.

Una vez que hemos visto las características generales de esta construcción y sus diferencias con respecto a otras construcciones interesa pasar a definir algunos conceptos relativos al cambio lingüístico y, en particular, los de construcción y construccionalización.

\section{CONSTRUCCIONALIZACIÓN Y CAMBIO LINGÜÍSTICO}

En los trabajos sobre cambio lingüístico se ha hablado de un cambio en la construcción, si bien no siempre se precise qué se entiende por construcción (Bisang, 1998: 13-15; Lehmann, 2002: 7-8; Hopper y Traugott, 2003: 142; Company, 2016: 389390). La gramática construccional es una teoría según la cual no se da una división entre léxico y gramática, sino que la diferencia entre ambos es de carácter gradual y más que un inventario meramente léxico tenemos almacenado un constructicón, esto es, una red estructurada de construcciones de distinta extensión y que pueden ser de carácter esquemático o sustantivo (Fillmore et al., 1988: 501; Goldberg, 2013, 1995: 1-7, 67-72; Traugott y Trousdale, 2013: 151). En esta teoría, por construcción se entiende que se trata de un signo, esto es, una estructura con una correspondencia entre forma y significado, con propiedades sintácticas, semánticas y pragmáticas (p. ej. Goldberg, 1995: 1-7). El proceso de cambio lingüístico implica tanto cambios construccionales como una construccionalización, por la que se entiende: 
Constructionalization is the creation of form ${ }_{\text {new }}$-meaning $_{\text {new }}$ (combinations of) signs. It forms new type nodes, which have new syntax or morphology and new coded meaning, in the linguistic network of a population of speakers. It is accompanied by changes in degree of schematicity, productivity, and compositionality (Traugott \& Trousdale, 2013: 22).

En la bibliografía especializada suele hablarse de dos procesos de cambio: la lexicalización y la gramaticalización (cf. p. ej. Lehmann, 1989, 2002; Giacalone Ramat, 1998: 120-122; Moreno Cabrera, 1998; Auwera, 2002; Brinton y Traugott, 2005: 62-88); al hablar de construccionalización se pueden abordar ambos tipos de cambio, considerando, como hemos señalado, que la diferencia entre léxico y gramática es de carácter gradual. Así, se habla de construcciones más léxicas, relacionadas con el contenido, y construcciones más gramaticales, relacionadas con el carácter procedimental de su significado (Traugott y Trousdale, 2013). Asimismo, se admite la existencia de construcciones híbridas (ibid.). Las construcciones de indefinición son, según nuestra hipótesis, una construcción de este último tipo, en el sentido de que indican un significado procedimental, el de indefinición, y señalan asimismo una diferencia de registro según se elija uno u otro verbo (QUERER/PREFERIR/CONSIDERAR/DARLE la gana etcétera). En el caso concreto de la construcción de indefinición con DAR la gana, muestra rasgos de haber pasado por un proceso de construccionalización, como hemos presentado en trabajos previos (Ruiz Velasco, en prensa): se inserta en un esquema constructivo, a saber, NEXO SUBORDINANTE + EXPRESIÓN VERBAL DE DISPOSICIÓN MENTAL; presenta un marcado aumento en su productividad, y su composicionalidad ha disminuido, como muestran los rasgos idiomáticos que hemos mencionado con anterioridad. En lo general, con la inserción del determinante definido la construcción se especializó para participar en las construcciones indefinidas; con respecto a la construcción de base, DAR + un sustantivo de afección (DAR miedo/pena/lástima/...), una comparación entre ambas muestra que en la actualidad DAR la gana forma una construcción distinta, con rasgos semánticos y sintácticos propios (cf. Ruiz Velasco, 2017).

Ahora bien, en los estudios de cambio lingüístico también se ha señalado la importancia del contexto. Heine (2002: 84-86) distingue cuatro tipos: el contexto inicial, en el que la forma aparece con un valor; el contexto puente, en el que cabe asignarle dos interpretaciones a la expresión en cuestión; el contexto de cambio, en el que la interpretación con el valor inicial es bloqueada, y los contextos de convencionalización, cuando se ha generalizado la segunda interpretación a otros contextos. De manera similar, Diewald (2006: 4-5) distingue 
tres tipos de contexto: el contexto atípico, en el que se dan las precondiciones para la gramaticalización mediante un sentido añadido como una implicatura conversacional; el contexto crítico, en el que inicia la gramaticalización propiamente dicha y se da una opacidad en el significado, y el contexto aislado, en el que se favorece una lectura a exclusión de la otra, se consolida la gramaticalización y la construcción deviene polisémica. En el proceso de cambio interviene, pues, la extracción de inferencias: en un primer momento se trata de una implicatura ad hoc para interpretar un enunciado, conforme se va generalizando y empleando en más contextos, dicha implicatura pasa a ser absorbida como parte del significado de la construcción en cuestión (Heine, 2002; Bybee, 2010: 171, y esp. Traugott y Dasher, 2005: 16-19, 34-40). En este sentido se ha hablado de ambigüedad, como un paso previo para el cambio lingüístico al permitir el reanálisis de la misma, y de polisemia, como la existencia de dos significados de una construcción en el nivel sincrónico.

En el caso de DAR la gana no se ha llegado a un contexto de convencionalización en el que la interpretación indefinida sea la única posible, sino que tiene carácter polisémico y coexiste el significado de indefinición con el volitivo. En lo que sigue hacemos una revisión tomando en cuenta primeramente la construcción DAR gana, pues el cambio parece originarse primeramente en dicha construcción y enseguida pasamos a revisar el caso de DAR la gana. Para ello distinguimos tres tipos de contexto: los contextos de asociación léxica con 'voluntad', los contextos con una implicatura de indefinición y los contextos de indefinición.

\section{LAS CONSTRUCCIONES DE INDEFINICIÓN Y EL CONTEXTO}

Los datos con los que trabajamos provienen del CORDE, en el que se hicieron búsquedas específicas para los siglos XVI al XIX. Los casos analizados ascienden a 644 para toda la familia de construcciones con gana ${ }^{1}$. Específicamente para la construcción DAR gana contamos con 131 casos, y para la construcción DAR la gana, 405. Para este trabajo se revisó cada uno de los ejemplos y se determinó cuáles entraban en el esquema de construcción indefinida, así como su contexto de uso.

${ }^{1}$ Dado que el corpus no está etiquetado para la consulta esquemática, se hicieron búsquedas específicas de "DAR gana" y las otras colocaciones en cada uno de los tiempos verbales simples, y para cada uno de los siglos. Este tipo de búsqueda es más eficiente con dicho corpus, pero tiene sus limitantes, ya que no permite ver si en algunos casos se daba algún elemento interpuesto, o la anteposición del sustantivo. 


\subsection{DAR gana}

Hemos señalado que la construcción de indefinición de que nos ocupamos tiene un carácter frasal, siguiendo la fórmula NEXO SUBORDINANTE + EXPRESIÓN VERBAL DE DISPOSICIÓN MENTAL. Ahora bien, no toda subordinación implica que se trata de una construcción indefinida. Veamos los ejemplos (1) y (2):

(1) ¿qué es la razón que si un hombre tiene deseo del acto carnal, ha vergüenza de manifestarlo, y si le da gana de comer, o beber, o de cualquier cosa de este género, no tiene empacho de manifestarlo? (s. XVI, Juan Huarte de San Juan, Examen de ingenios para las ciencias).

(2) Yo os digo, hermanas, que cuando se me acuerda el miedo de mi compañera, que era María del Sacramento -una monja de más edad que yo y harto sierva de Dios-, que me da gana de reir (s. XVI, Santa Teresa de Jesús, Libro de las fundaciones)

En ambos casos no tenemos construcciones indefinidas. En ambos influye el hecho de que se tenga un complemento oracional introducido por de: en (1) gana de comer, o beber, o de cualquier cosa de este género; en (2) gana de reir. Para ser una construcción indefinida se requiere de un sintagma escueto, como mencionamos en el apartado 1 . Asimismo, interviene el tipo de construcción subordinada introducida por que: en el primer caso introduce una oración sustantiva, las cuales no suelen tener una interpretación indefinida ${ }^{2}$; en el segundo, se trata de un que explicativo, el cual tampoco introduce las construcciones de indefinición de que nos ocupamos. Para ser una construcción indefinida se requiere que el que participe en una construcción de relativo o una de las locuciones adverbiales del tipo siempre que, hasta que (cf. Ruiz Velasco, ms.). El primer ejemplo al que se le puede dar una lectura indefinida lo encontramos ya en el siglo XVI:

(3) Arnaldo [...] Quien tiene tanto cuidado / de honra y della se precia, / dexar sola ansí a Lucrecia / a sido mal acordado; / moça hermosa y galana, / y con tal vieja parlando, / ¿qué podrán estar tratando?

Albina De lo que les diere gana (s. XVI, Joaquín Romero de Cepeda, Comedia salvaje).

${ }^{2}$ En Ruiz Velasco (en prensa) citamos el único caso que encontramos al que se le puede dar una lectura indefinida. 
En este caso tenemos una relativa semilibre con lo que, con un sintagma escueto, y se le puede dar la lectura de cualquier cosa. El hablante (Albina) destaca la inespecificidad del contenido. De acuerdo con Haspelmath (1997: 46) los definidos indican que se trata de información conocida para hablante y oyente, mientras que los indefinidos señalan información desconocida para el oyente, pero varían en cuanto a si es conocida para el hablante o no. En el caso de (3) parece ser que el asunto tratado es desconocido tanto para el hablante (Albina) como para el oyente (Arnaldo). Cabe considerar este como el contexto sintáctico inicial, en el que se invita la implicatura indefinida.

Para el siglo XVII encontramos otro caso al que se le puede dar una lectura indefinida (4). En ese mismo siglo se documentan las primeras instancias de DAR la gana, las cuales ya tienen ese carácter indefinido. Para los siglos XVIII y XIX los casos son más numerosos. Damos un ejemplo de cada siglo, con DAR gana:

(4) Que se asome a la ventana / la vez que le diere gana, / y se huelgue y se solace, / que me place (s. XVII, Anónimo, Segunda parte del Romancero general y Flor de diversa poesía).

(5) a título de bufón dice cuanto le da gana (s. XVIII, Ramón de la Cruz, El Petimetre).

(6) Entonces nada queda que no le escudriñemos. Patria, padres, parentela, conducta, relaciones, y cuanto nos da gana, todo se sujeta á nuestras averiguaciones é inspeccion (s. XIX, Fray Francisco Alvarado, Cartas críticas del Filósofo Rancio, II).

Cabe señalar que en (6) el hablante emplea la expresión tras una enumeración y cierra con la expresión y cuanto nos da gana como recurso estilístico para evitar ser más específico; a diferencia de los ejemplos anteriores, en este caso, la información es desconocida para el oyente, que ha de imaginar el posible complemento, mas accesible para el hablante, esto es, para el nosotros del enunciado. Esta es una cualidad de la construcción: la información se presenta como accesible para el experimentante, mas inaccesible para entidades externas al tratarse de un proceso mental, de ahí que se preste para comunicar la indefinición.

Tenemos, pues, que con DAR gana ya existe el antecedente de enunciados con una lectura indefinida. Ello no quiere decir que sea su única función, ya que DAR gana es una construcción que se mantendrá ambigua oscilando entre un significado más cercano a DAR ganas y otro más cercano a DAR la gana. Ésta última en cambio se especializará en participar en las construcciones indefinidas (cf. Ruiz Velasco, en prensa). 


\subsection{DAR la gana}

Para DAR la gana distinguimos tres tipos de contextos: aquellos en los que se da una asociación léxica con palabras que apuntan al sentido de 'voluntad'; aquellos en que la lectura indefinida es ambigua y puede o no darse, y aquellos en que la lectura indefinida es clara en el contexto. Veamos cada uno por separado.

\subsubsection{Asociación léxica}

Hemos señalado que la construcción DAR la gana tiene dos valores, uno modal y otro más gramatical, como marca de indefinición en las construcciones indefinidas. Interesa en este apartado su valor modal en el que el significado de 'voluntad' de gana aparece en el primer plano discursivo. Para ello, cabe observar primero el significado léxico del sustantivo gana. El término mismo tiene carácter polisémico a partir de un desarrollo por el que fue ampliando su significado.

Una revisión del Nuevo Tesoro Lexicográfico de la Lengua Española (NTLLE) nos muestra que ya Nebrija definía gana en 1495 como «antojo» o «líbido», definición que es retomada por diversos autores. En los diccionarios de Percival de 1591 y de Palet de 1604 gana es traducido como «will, desire, lust» y «enuie, desir, volonté». En ellos encontramos por primera vez la equivalencia de gana con 'deseo' o 'voluntad'. Oudin en 1607 incluye además la equivalencia de «caprice». En la tradición hispánica, tales equivalencias las encontramos hasta el diccionario de Covarrubias de 1611, cuya entrada señala: «vale deseo, apetito, voluntad, y aquellas cosas de que tenemos gana las apetecemos, por tener gozo y contento enellas, y assi trae origen esta palabra del nombre Griego $\gamma \dot{\alpha} v s$, ganos, que vale tanto como gozo, y còtento». En el tomo IV (1734) del Diccionario de autoridades de la Academia se da como equivalentes «Deséo, apetíto, propensión, voluntad, inclinación de alguna cosa: como de comer, dormir, \&c.», definición que con ligeras variaciones se mantiene hasta el diccionario de 2001. Cabe aclarar que la identificación de gana con el significado de «deseo o propensión natural» se empleará más con la construcción DAR ganas y la de «voluntad» con la de DAR la gana (cf. Ruiz Velasco, en prensa).

Ahora bien, en el discurso puede ser que la construcción DAR la gana, aun siguiendo el esquema de las construcciones de indefinición a que hemos hecho referencia, conserve el sentido de 'voluntad' o 'disposición mental', esto es, mantenga su significado léxico. Ello es evidente cuando en el discurso co-aparece con una serie de lexemas asociados conceptualmente vía relaciones de similitud o sinonimia. Entre tales lexemas encontramos el de voluntad, así como los de antojo o libertad, además 
de otros más marcados axiológicamente como desvergüenza o anarquí, y expresiones frasales con un sentido similar. Damos un par de ejemplos:

(7) a. Todos los géneros encarecen, y pujan más por abarcarlos, y con eso, siendo suyos, los venden en Manila al precio de su voluntad; y así se les da bien poco de comprarlos más caros en estas provincias, porque saben que allá en Manila los venderán á como les diere la gana (s. XVIII, Juan José Delgado, Historia general sacro-profana, politica y natural del Poniente llamadas Filipinas).

b. Después van a Segovia, donde Villamil se propone pintar la Fuencisla, el Parral, y qué se yo qué, y mi marido ver y tasar una colección de clavos de puertas, bisagras y aldabones que a la venta sale. Por allá se están luegos días, y si fueran meses, mejor, para que yo respire. jPreciosa libertad, cuánto vales! Así podré llorar a mis anchas a mi amada Justina, y llevarle flores, y hablar contigo, emborronando todo el papel que me dé la gana. ¡Benditas cacerías de la Encomienda y benditos clavos de Segovia! Claro que mi libertad sólo es relativa, porque siempre quedan aquí personas que al volver Felipe le cuentan todo lo que hago; pero esta clase de esclavitud la sorteo yo perfectamente (s. XIX, Benito Pérez Galdós, La estafeta romántica).

En ambos ejemplos tenemos un contexto discursivo en el que se resalta el valor léxico de gana. Estos contextos se mantienen hasta el siglo XIX, como muestra el último ejemplo, proveniente de Benito Pérez Galdós.

\subsubsection{Implicatura de indefinición}

Hemos señalado que, dentro del desarrollo diacrónico, ciertos contextos sirven de puente, en el sentido de que mantienen una lectura ambigua en la que se puede entender tanto la lectura inicial de una construcción como una posterior. En el caso que nos ocupa, los contextos que sirven de puente corresponden a contextos en los que DAR la gana señala un conjunto indefinido, si bien la lectura de 'voluntad' también es posible. Ello se hace evidente cuando en el discurso es claro que se trata de una opción entre otras:

(8) a. Ella. Qué señorita buscas / saber pretendo.

El. La que me da la gana (s. XVIII, José Subirá, Yo soy una majota. Tonadilla a dúo). 
b. -Error obeso se dice. Sé bien parlado —advirtió Botaña, con una risotadita.

-Obeso, adiposo o como te dé la gana, Cervantes... (s. XIx, Emilia Pardo Bazán, El niño de Guzmán).

En los ejemplos ( $8 \mathrm{a}$ y b) va aparejada la implicatura de que podría equivaler simplemente a sea: la que sea, como sea, una lectura que puede coexistir con la de 'voluntad'. Este tipo de implicaturas pueden cancelarse, como ocurre en el siguiente ejemplo al añadirse «siempre y cuando lo halle por conveniente»:

(9) puede V. libremente, sin que nadie le contradiga, antes bien con aplauso, recomendación y elogio de los escritores liberales, traer, no solo á Dios y á su religion, que eso es poco, mas también á santo Tomás y á cualquier teólogo del siglo XIII, á Mariana del XVI, y á todo el que le dé la gana, siempre y cuando lo halle por conveniente (s. XIX, Fray Francisco Alvarado, Cartas críticas del Filósofo Rancio, II).

\subsubsection{Indefinición}

En los contextos de indefinición, esta es la única interpretación posible. Se trata de los llamados contextos de cambio, en la descripción de Heine (2002), o contextos aislados, en la descripción de Diewald (2006). En ellos queda bloqueada la interpretación con el significado de 'voluntad'. El cambio se observa cuando la construcción es empleada con eventos inagentivos, que por su significado bloquean la lectura de 'voluntad', como ocurre con un verbo como llamarse:

(10) No hablemos más de ello, padre mío; que Melián vaya bendito de Dios a corregir indios a Nicaragua y que la hija de Girón se llame Sor Margarita o como le dé la gana (s. XIX, José Milla y Vidaurre, El visitador).

Asimismo, en el CORDE encontramos casos en que la paráfrasis que se presenta posteriormente hace claro el sentido de elección libre y no el de 'voluntad'. En (11) se presenta primeramente una canción:

(11) Sin que la rueda pare, / desde ahora se manda / que cada una se abrace / con quien le dé la gana (s. XIX, Fausto López Villabrille, Colección de juegos para niños de ambos sexos). 
Para explicar enseguida:

(12) Concluido esto, se ejecuta lo expresado en los dos últimos versos: las niñas (que tienen que ser en número impar) se abrazan con la primera que hallan (s. XIX, Fausto López Villabrille, Colección de juegos para niños de ambos sexos).

Estos usos indican que se está dando un proceso de desemantización ${ }^{3}$. Cabe considerar que una consecuencia de ello es que en ciertas variedades del español se haya optado por el renovamiento de la construcción mediante el cambio del núcleo verbal. Así, surgen las variantes PEGÁRSELE la gana e HINCHÁRSELE la gana.

\section{CONCLUSIONES}

Las construcciones de indefinición con DAR la gana tienen un carácter menos gramaticalizado que el de los compuestos indefinidos como cualquier(a). Siguiendo los parámetros clásicos de la gramaticalización, el carácter más fusionado de tales compuestos sería ya un indicador de ese tipo, pero también lo es su semántica: se interpretan directamente con un contenido indefinido, si bien hayan dado pie también a otros significados (cf. Company y Pozas, 2009). En el caso de las estructuras frasales de que nos ocupamos, no se ha alcanzado un significado único, sino que encontramos una suerte de gradiente del significado. Este rasgo del significado de la construcción lo podemos ver en su carácter polisémico: la construcción es empleada tanto con un sentido más volitivo como con un sentido claro de indefinición. El proceso de cambio, como hemos mostrado, se inicia con la construcción DAR gana en la que el sentido de indefinición comienza a aparecer al emplearse en entornos sintácticos particulares: subordinadas de relativo y con un sintagma escueto. Podría añadirse el hecho de emplearse el modo subjuntivo (ejemplos 3 y 4), sin embargo, los datos de que disponemos no nos permiten afirmarlo en forma categórica, sí en cambio los dos rasgos que hemos mencionado ${ }^{4}$. Ya en las construcciones con DAR la gana se constata el carácter polisémico de la construcción: un contexto discursivo en el que aparecen lexemas ligados al significado de 'voluntad' trae a primer plano dicha interpretación volitiva aun cuando no quede descartada la interpretación in-

${ }^{3}$ Los ejemplos orales del Corpus de Referencia del Español Actual (CREA) presentan una variación similar a la reseñada aquí: casos claros; casos con una lectura ambigua, y casos en que se presentan asociaciones léxicas con 'voluntad' como recurso retórico, de ahí que consideremos que el valor de indefinición no ha alcanzado la suficiente convencionalización.

${ }^{4}$ Para ello remitimos al lector tanto a los datos aquí presentados como en trabajos previos (Ruiz Velasco, en prensa y ms.). 
definida; contextos sin esa carga léxica traen a primer plano la lectura polisémica en los que ambas interpretaciones son posibles si bien, como muestra el ejemplo (9), la lectura indefinida puede ser cancelada en el contexto posterior; finalmente, ciertos contextos a los que se ha extendido el uso de la construcción bloquean una lectura 'volitiva'. Lo anterior confirma el carácter gradiente del significado que ha sido planteado en teorías gramaticales basadas en el uso (cf. Bybee, 2010).

Por último, no está de más resaltar el hecho de que estas construcciones permiten una lectura no específica que abarca tanto contextos en los que hablante y oyente desconocen la información como aquellos en los que la información es desconocida para el oyente mas no para el hablante. La instrucción que presentan al oyente, su significado procedimental, es meramente que la información es desconocida para este y, por tanto, es de elección libre.

\section{BIBLIOGR AFÍA}

\section{Fuentes primarias}

Covarrubias, Sebastián de (1611): Tesoro de la lengua castellana o española, Madrid, Luis Sánchez.

Nebrija, Antonio de (1989[1495]): Vocabulario español-latino (edición facsimilar), Madrid, Real Academia Española.

Oudin, César (1607): Tesoro de las dos lenguas francesa y española. Thresor des deux langues françoise et espagnolle, París, Marc Orry.

PALET, Juan (1604): Diccionario muy copioso de la lengua española y francesa [...]. Dictionaire tres ample de la langue espagnole et françoise, París, Matthieu Guillemot.

Percival, Richard (1591): Bibliothecae Hispanicae pars altera. Containing a Dictionarie in Spanish, English and Latine, Londres, John Jackson y Richard Watkins.

Real Academia Española (1734): Diccionario de la lengua castellana, en que se explica el verdadero sentido de las voces, su naturaleza y calidad, con las phrases o modos de hablar, los proverbios o refranes, y otras cosas convenientes al uso de la lengua [...]. Compuesto por la Real Academia Española. Tomo quarto. Que contine las letras G.H.I.J.K.L.M.N, Madrid, imprenta de la Real Academia Española, por los herederos de Francisco del Hierro.

Real Academia Española: Corpus diacrónico del español (CORDE). En línea: $<$ http://corpus.rae.es/cordenet.html> [16/04/2017]. 
Real Academia Española: Nuevo Tesoro Lexicográfico de la Lengua Española. En línea: <http://ntlle.rae.es/> [14-15/03/2019].

\section{Fuentes secundarias}

AuwerA, Johan van der (2002): «More thoughts on degrammaticalization», en Ilse Wischer y Gabriele Diewald (eds.), New reflections on grammaticalization, Amsterdam y Philadelphia, John Benjamins, pp. 19-29.

BIsANG, Walter (1998): «Grammaticalization and language contact, constructions and positions», en Anna Giacalone Ramat y Paul J. Hopper (eds.), The limits of grammaticalization, Amsterdam y Philadelphia, John Benjamins, pp. 13-58.

Brinton, Laurel J. y Elizabeth Closs TraugotT (2005): Lexicalization and language change, Cambridge, Cambridge University Press.

Bybee, Joan (2010): Language, usage and cognition, Cambridge, Cambridge University Press.

Company Company, Concepción (2016): «Sintaxis histórica y tradiciones discursivas. El género textual como macrolocus del cambio sintáctico», en Araceli López Serena, Antonio Narbona Jiménez y Santiago del Rey Quesada (dirs.), El español a través del tiempo. Estudios ofrecidos a Rafael Cano Aguilar, Sevilla, Universidad de Sevilla, vol. 1, pp. 385-413.

Company Company, Concepción y Julia Pozas Loyo (2009): «Los indefinidos compuestos y los pronombres genérico-impersonales omne y uno», en Concepción Company Company (dir.), Sintaxis histórica de la lengua española. Segunda parte: La frase nominal, México, UNAM y FCE, vol. 2, pp. 1073-1219.

Diewald, Gabriele (2006): «Context types in grammaticalization as constructions», Constructions, 1, 9, pp. 1-29.

FERnández Ramírez, Salvador (1987): Gramática española. 3.2. El pronombre, Madrid, Arco-libros.

Fillmore, Charles J. et al. (1988): «Regularity and idiomaticity in grammatical constructions: The case of let alone», Language, 64, 3, pp. 501-538.

Goldberg, Adele (1995): Constructions. A construction grammar approach to argument structure, Chicago y London, University of Chicago Press.

Goldberg, Adele (2013): «Constructionist approaches», en Thomas Hoffmann y Graeme Trousdale (eds.), The Oxford Handbook of Construction Grammar, Oxford, Oxford University Press, pp. 15-31.

Haspelmath, Martin (1997): Indefinite pronouns, Oxford, Oxford University Press. 
HeINE, Bernd (2002): «On de role of context in grammaticalization», en Ilse Wischer y Gabriele Diewald (eds.), New reflections on grammaticalization, Amsterdam y Philadelphia, John Benjamins, pp. 83-101.

Hopper, Paul J. y Elisabeth Closs TraugotT (2003): Grammaticalization, 2. ${ }^{\text {ed., }}$ Cambridge, Cambridge University Press.

Lehmann, Christian (1989): «Grammatikalisierung und Lexikalisierung», Zeitschrift für Phonetik, Sprachwissenschaft und Kommunikationsforschung, 42, pp. 11-19 (2016, en línea: <http://www.christianlehmann.eu/publ/lehmann_lexikalisierung.pdf> [08/05/2017].

LeHmanN, Christian (2002): «New reflections on grammaticalization and lexicalization», en Ilse Wischer y Gabriele Diewald (eds.), New reflections on grammaticalization, Amsterdam y Philadelphia, John Benjamins, pp. 1-18.

LeOnetti, Manuel (1999): «El artículo», en Ignacio Bosque y Violeta Demonte (dirs.), Gramática descriptiva de la lengua española, Madrid, Espasa Calpe, vol. I, pp. 787-890.

Lyons, Christopher (1999): Definitenes, Cambridge, Cambridge University Press. Moreno Cabrera, Juan C. (1998): «On the relationship between grammaticalization and lexicalization», en Anna Giacalone Ramat y Paul J. Hopper (eds.), The limits of grammaticalization, Amsterdam y Philadelphia, John Benjamins, pp. 211-227.

Ramat, Anna Giacalone (1998): «Testing the boundaries of grammaticalization», en Anna Giacalone Ramat y Paul J. Hopper (eds.), The limits of grammaticalization, Amsterdam y Philadelphia, John Benjamins, pp. 107-127.

Ruiz Velasco D., Liliana (2017): «Porque me da la gana o la razón de la sinrazón: un análisis construccional», Lingüistica Mexicana, 9, 1, pp. 19-42.

Ruiz Velasco D., Liliana (en prensa): «Desarrollo sintáctico de una familia de construcciones con gana y fijación de las construcciones de indefinición con DAR la gana», Nueva Revista de Filología Hispánica.

Ruiz Velasco D., Liliana (2019) (ms.): «Las construcciones de indefinición con DAR la gana $\gg$.

TraugotT, Elizabeth Closs y Richard B. Dasher (2005): Regularity in semantic change, Cambridge, Cambridge University Press.

TraugotT, Elizabeth Closs y Graeme Trousdale (2013): Constructionalization and constructional changes, Oxford, Oxford University Press. En línea: <www. oxfordscholarship.com> [18/01/2019].

Fecha de recepción: 7 de mayo de 2019 Fecha de aceptación: 24 de septiembre de 2019 
